\title{
Fahrerlose Shuttles im öffentlichen Personennahverkehr: Akzeptanz und Einstellung in der Bevölkerung
}

\author{
Mathias Wilde' • Jakob Rebhan ${ }^{1}$ \\ Eingegangen: 7. Mai 2021 / Überarbeitet: 13. September 2021 / Angenommen: 28. September 2021 / Online publiziert: 25. Oktober 2021 \\ () Der/die Autor(en) 2021
}

\section{Zusammenfassung}

Aktuell erproben einige Modellprojekte sowohl die Technik als auch den Betrieb fahrerloser Fahrzeuge im öffentlichen Verkehr. Eines davon ist die Shuttle-Modellregion Oberfranken. Um Aussagen zu den Einstellungen in der Bevölkerung generieren zu können, wurde eine Akzeptanzstudie durchgeführt. Im Ergebnis zeigte sich, dass bereits vor dem Betriebsbeginn eine ausgeprägte Nutzungsbereitschaft besteht. Offenkundig auch eine überhöhte Erwartungshaltung: Mit dem Einsatz fahrerloser Shuttles erwarten die Menschen flexible Abfahrten und eine hohe Taktdichte, Pünktlichkeit und neue Strecken, bessere Erreichbarkeit und Angebote in Randzeiten. Diesen Anforderungen kann die aktuelle Fahrzeuggeneration noch nicht gerecht werden. Insofern ist es angebracht, ein besonderes Augenmerk auf die symbolische Aufladung zu legen. Eine Diskrepanz zwischen gesellschaftlichem Narrativ, persönlicher Erwartungshaltung und gegebener Leistungsfähigkeit der Fahrzeuge könnte die Akzeptanz gefährden.

Schlüsselwörter Autonome Busse · Nutzerakzeptanz · Autonomes Fahren · Automatisierter Personentransport

\section{Driverless shuttles in local public transport: acceptance and attitudes among the population}

\begin{abstract}
A variety of case studies are currently testing both the technology and the operation of driverless vehicles in public transport. One of these is the "Shuttle-Modellregion Oberfranken". In order to gain insights into the attitudes of the people, an acceptance study was conducted. The results indicate that there is a pronounced willingness to use the system, but obviously excessively high expectations too: with the use of driverless shuttles, people expect flexible departures and a high frequency, punctuality and new lines, better accessibility and services at off-peak times. The current generation of vehicles is unable to meet these expectations. For this reason, it is necessary to pay particular attention to the socio-cultural expectations. A discrepancy between the societal narrative, personal expectations and the given performance of the vehicles could compromise this acceptance.
\end{abstract}

Keywords Self-Driving Shuttle $\cdot$ User acceptance $\cdot$ Autonomous vehicles $\cdot$ Public driverless transport

Mit dem Einsatz von fahrerlosen Shuttles im öffentlichen Personennahverkehr (ÖPNV) ist die Hoffnung verbunden, das bestehende Verkehrsangebot zu ergänzen und Leistungen auch bei geringer Nachfrage betreiben zu können. Ei-

Prof. Dr. Mathias Wilde

mathias.wilde@hs-coburg.de

1 Fakultät Maschinenbau und Automobiltechnik, Hochschule für angewandte Wissenschaften Coburg, Friedrich-Streib-Str. 2, 96450 Coburg, Deutschland ne besondere Herausforderung ist neben Technik, Planung und Betrieb die Akzeptanz der Bevölkerung. Der Erfolg ist maßgeblich abhängig vom Vertrauen in das System.

Inzwischen suchen zahlreiche Modellprojekte nach Antworten auf Fragen zum Betrieb fahrerloser Shuttles. Eines davon ist die Shuttle-Modellregion Oberfranken (SMO), zu der sich die Kommunen Hof, Rehau und Kronach zusammengeschlossen haben (Abb. 1).

In der Modellregion führte die Hochschule Coburg vor Aufnahme des Shuttlebetriebs eine Akzeptanzstudie durch. Sie hatte zum Ziel, die Akzeptanz und Nutzungsbereitschaft 
Abb. 1 Fahrerlose Shuttles des SMO-Projektes - in Hof (a) und Kronach (b). (Quelle: Stadt Hof, Landkreis Kronach)
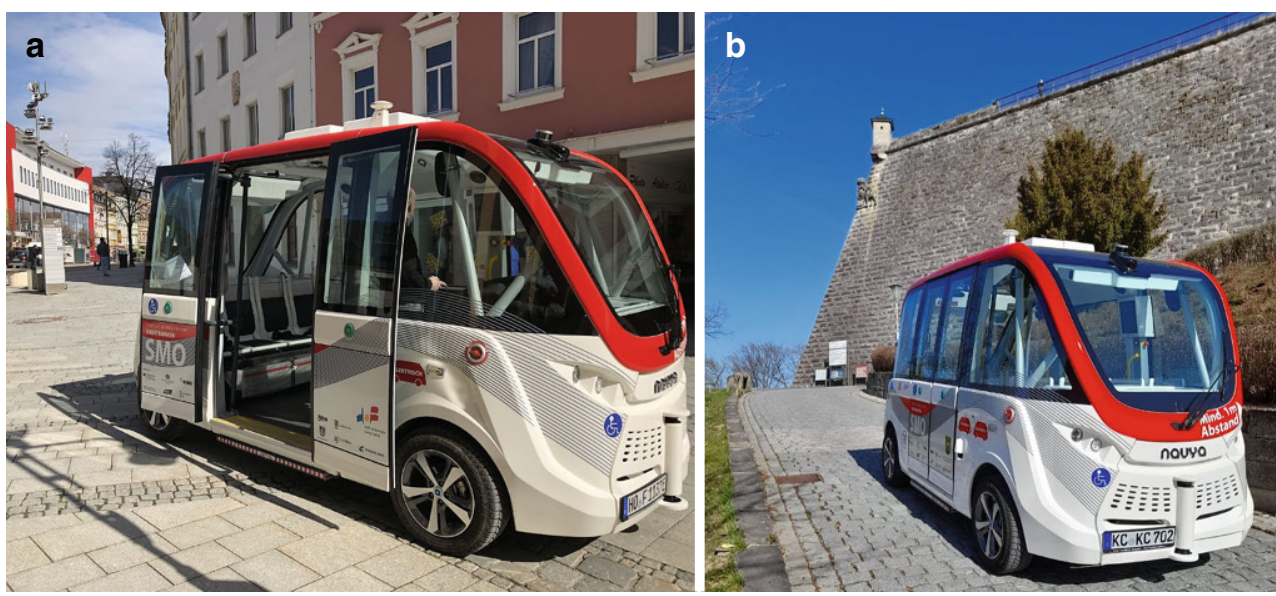

von fahrerlosen Shuttles in der Bevölkerung abzubilden. Im Ergebnis konnten Aussagen zu den Einstellungen, Wertzuschreibungen und Anforderungen an einen Shuttlebetrieb generiert werden. Ein weiteres Ziel lag in der Ausarbeitung eines Akzeptanzmodells, das die Rahmenbedingungen der Nutzungsintention abbildet.

\section{Stand der Forschung}

Neben der Nutzungsbereitschaft thematisiert die Akzeptanzforschung zu fahrerlosen Shuttles die Sicherheit, den Komfort und die Geschwindigkeit als vermittelnde Faktoren. Deren Ergebnisse verweisen auf eine hohe Nutzungsintention (Bernhard et al. 2020; Rauh et al. 2020). Vertrauen in die Technik wird als eine entscheidende Akzeptanzgröße angesehen (vgl. Paddeu et al. 2020). Kassens-Noor et al. (2020) kommen zu dem Ergebnis, dass die Hälfte derjenigen ebenfalls fahrerlose Shuttles nutzen würden, die bereits regelmäßig mit dem klassischen ÖPNV unterwegs sind, wobei mehr jüngere Menschen als ältere dem fahrerlosen System positiv gegenüber eingestellt sind. Insbesondere in der Sicherheit und Zuverlässigkeit finden sich Kriterien, die auf die Nutzungsintention einwirken (Chen 2019). Dabei kann man einerseits unterscheiden zwischen der gefahrlosen Verkehrsteilnahme der Fahrzeuge und dem Sicherheitsempfinden der Fahrgäste im Innenraum andererseits. Ein hohes Sicherheitsempfinden während der Fahrt ist ausschlaggebend dafür, ob die Menschen bereit sind, auch ohne Begleitpersonal die Shuttles zu nutzen (Rosell und Allen 2020).

Studien zur Akzeptanz von automatisierten Fahrsystemen im Allgemeinen (vgl. Nordhoff et al. 2020) und im Speziellen zu fahrerlosen Shuttles (vgl. Bernhard et al. 2020) fundieren ihre Arbeit oft mit der „Unified Theory of Acceptance and Use of Technology (UTAUT)“ (Venkatesh et al. 2003) und passen sie an ihre Anforderungen an. Während diese Ansätze die individuelle Ebene gut abbil- den, vernachlässigen sie die gesellschaftliche Dimension. An dieser Stelle greifen die Überlegungen von Lenz und Fraedrich (2015), sie heben die sozial-kulturellen Aspekte hervor und verweisen darauf, dass sowohl das Individuum als auch das Akzeptanzobjekt im Kontext stehen zur Gesellschaft, der Situation und dem Zeitpunkt.

\section{Vorgehen und Methode}

Bei der vorliegenden Studie handelt es sich um eine standardisierte Haushaltsbefragung. Die abgefragten Items orientieren sich an den oben skizzierten Ansätzen der Technikakzeptanz. Die Befragung richtete sich an die Bevölkerung der 3 Kommunen Hof, Rehau und Kronach im Regierungsbezirk Oberfranken/Bayern. Die regionalstatistische Raumtypologie kategorisiert die Orte als städtischen Raum (Rehau), Mittelstadt (Kronach) und zentrale Stadt (Hof) in einer peripheren ländlichen Region.

\section{Datenerhebung}

Die Befragung fand im September und Oktober 2020 statt und war als Onlinefragebogen konzipiert. Sie richtete sich an die 72.800 Menschen, die in den knapp 36.000 Haushalten der 3 Kommunen leben. Die Umfrage wurde über Aushänge und Handzettel, die Lokalpresse und soziale Medien sowie über die Kommunikationskanäle der Kommunen beworben. Der Fragebogen umfasste 66 Items, die in $3 \mathrm{Ka}-$ tegorien untergliedert sind:

1. Erwartungen und Meinungen: Die Kategorie zu den Erwartungen fragte nach den Ansichten zur Technik und den Einsatzszenarien. Ergänzend gingen Fragen etwa zur Wunschgeschwindigkeit oder zur Bewertung von Vorund Nachteilen ein.

2. Erfahrung mit Fahrerassistenzsystemen im Pkw: Abgefragt wurden Erfahrungen zu unterstützenden Systemen 


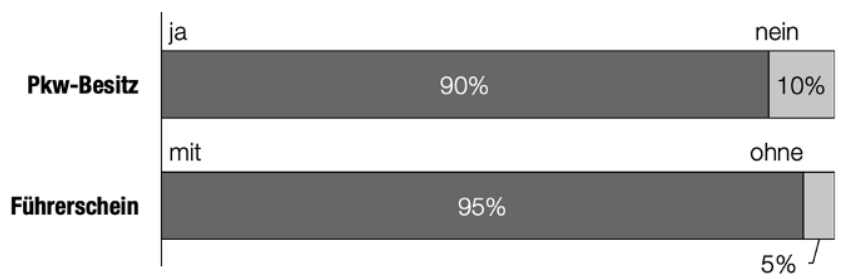

Abb. 2 Besitz von Pkw und Führerschein der Stichprobe

im Individualverkehr, die Fahrfunktionen übernehmen und automatisiert Entscheidungen im Verkehr treffen. Die Antworten gehen in die Beurteilung zur Technikakzeptanz ein.

3. Persönlichkeitsmerkmale: Neben soziodemografischen Angaben wie Alter, Geschlecht und Einkommen sind Fragen zum Mobilitätsverhalten und der Verkehrsmittelnutzung enthalten.

\section{Stichprobe}

Es ergab sich ein Rücklauf von 542 vollständigen Fragebögen. Mit Einschränkungen bei der sehr jungen und älteren Gruppe zeigte sich eine relativ homogene Altersverteilung. Die Befragten weisen sowohl einen relativ hohen Pkw- als auch Führerscheinbesitz auf (Abb. 2).

\section{Akzeptanz und Nutzungsbereitschaft fahrerloser Shuttles}

Dem Ziel folgend, die Akzeptanz und Nutzungsbereitschaft einschätzen zu können, gliedert sich die Ergebnisdarstellung in 4 Kategorien. Hierbei verläuft die wesentliche Unterscheidung einerseits entlang individueller Merkmale sowie andererseits entlang von Kriterien auf gesellschaftlicher Ebene. Beide Dimensionen sollen jene Facetten veranschaulichen, die das Bild fahrerloser Shuttles in der Bevölkerung wiedergeben.

\section{Wissen und Erfahrungen}

Wissen und Erfahrungen sind auf Ebene des Individuums verortet. Sie geben Einblick in persönliche Erwartungen und Bewertungen. Dafür wurde nach der ÖPNV-Nutzung und dem Wissen um alternative Mobilitätskonzepte gefragt - sie liefern Hinweise auf die generelle Einstellung zu öffentlichen ÖPNV-Angeboten. Demgegenüber bildet die Akzeptanz von bestehenden Fahrerassistenzsystemen die prinzipielle Bereitschaft ab, die Kontrolle an technische Hilfsmittel abzugeben.

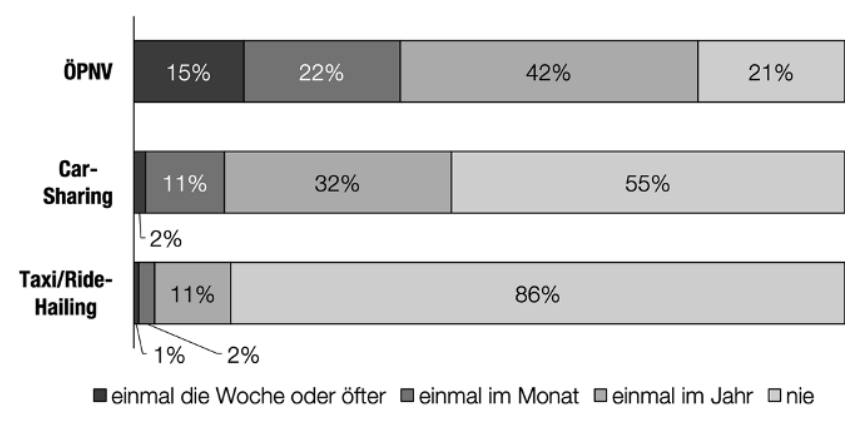

Abb. 3 Verkehrsmittelnutzung

ÖPNV und alternative Mobilitätskonzepte Die ÖPNV-Nutzung ist für einige der Befragten eine feste Größe in der Alltagsmobilität: $15,5 \%$ nutzen den ÖPNV einmal die Woche oder öfter, weitere $22 \%$ sind einmal im Monat mit dem ÖPNV unterwegs (Abb. 3).

Jenseits des klassischen ÖPNV sind alternative Angebote wie Mitfahrgelegenheit und Carsharing den Befragten zwar ein Begriff, genutzt werden sie allerdings weniger. Wobei sich die geringe Nutzung mit dem mäßig ausgeprägten Angebot in der Region erklären lässt.

Im geringen Angebot kann auch ein Grund für die fehlende Bekanntheit alternativer Mobilitätskonzepte liegen. So sind die Befragten mit multimodalen Dienstleistungen kaum vertraut.

Fahrerassistenz Seit einigen Jahren werden Fahrerassistenzsysteme in Kraftfahrzeuge verbaut. Eine Mehrheit der Befragten $(68,5 \%)$ nutzt regelmäßig Fahrerassistenzsysteme auf eigenen Fahrten. Daraus lässt sich zunächst die Vermutung ableiten, dass zumindest eine Teilgruppe mit jener Fahrzeugtechnik vertraut sein dürfte, die in das Fahrverhalten eingreift und dem Menschen Fahrfunktionen abnimmt. Dieser mögliche Zusammenhang, aber auch die Frage inwieweit Erfahrungen mit Fahrerassistenzsystemen im Pkw die Erwartungshaltungen beeinflussen, kann Ausgangspunkt weiterer Studien sein.

\section{Shuttlebetrieb: Erwartung und Bewertung}

Im Zuge der Einführung neuer Technik spielt Vertrauen eine einflussreiche Rolle. Vertraut die Bevölkerung der Technik und erfüllt das Angebot deren Erwartung, werden fahrerlose Shuttles eher angenommen.

Vertrauen und Einschätzung der Technikreife Bezüglich der Einschätzung zur Reife der Technik und Sicherheit der Fahrzeuge sowie des Vertrauens in das Begleitpersonal und die Ingenieure des Herstellers streuen die Ergebnisse stark. Zwar gibt ein Großteil an, die Technik für ausgereift zu halten, demgegenüber findet sich wiederum ein Teil, der der Technik skeptisch gegenübersteht. Insgesamt ist das Ver- 


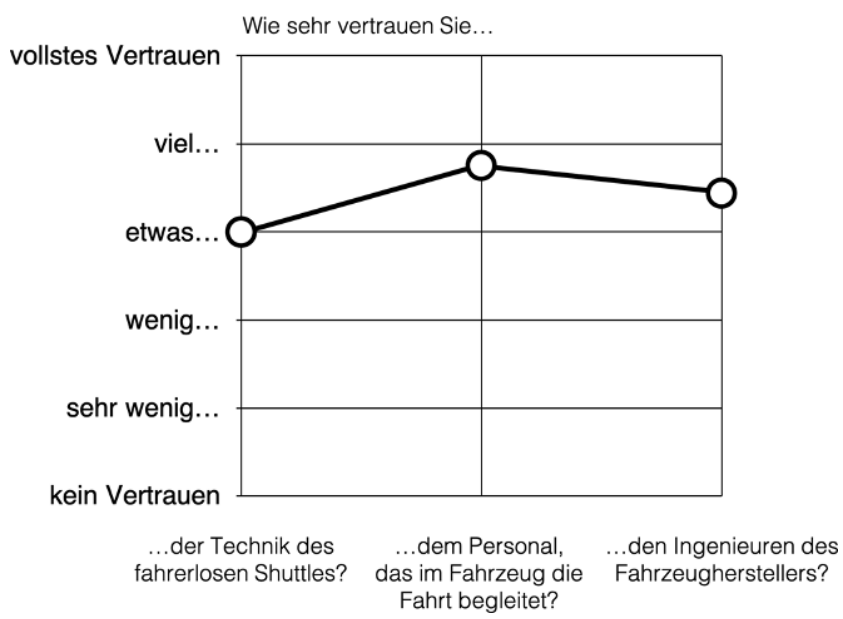

Abb. 4 Einschätzung zum Vertrauen in Bezug auf Technik und Personal

trauen in das fahrtbegleitende Personal und die Ingenieure größer als das in die Technik der Fahrzeuge (Abb. 4).

Eine offene Frage nach den Vorteilen wurde mit Aussagen beantwortet wie: „Mögliche Erhöhung der Frequenz des Nahverkehrs“, „Mehr Flexibilität durch kürzere Intervalle bei der Anfahrt der Haltestellen, langfristig Kostenersparnis, mehr Mobilität für ältere und Menschen ohne Fahrzeug“ oder „Der Bus kann immer fahren - egal zu welcher Uhrzeit".

Mitfahrt Generell sind die Befragten positiv gegenüber dem Betrieb eingestellt. Die höchste Zustimmung erhält die Aussage, dass man neugierig sei auf eine Fahrt mit einem fahrerlosen Shuttle. Außerdem überwiegt die Meinung, mit einem fahrerlosen Shuttle sicher an das Ziel zu gelangen (Abb. 5). Demgegenüber bestehen Zweifel, vor allem was Effekte auf den übrigen Verkehr anbelangt. Hier wird vor allem die Geschwindigkeit als unzureichend bewertet.

Geschwindigkeit Die derzeitigen $18 \mathrm{~km} / \mathrm{h}$ Höchstgeschwindigkeit und die teilweise deutlich darunter liegende Durch-

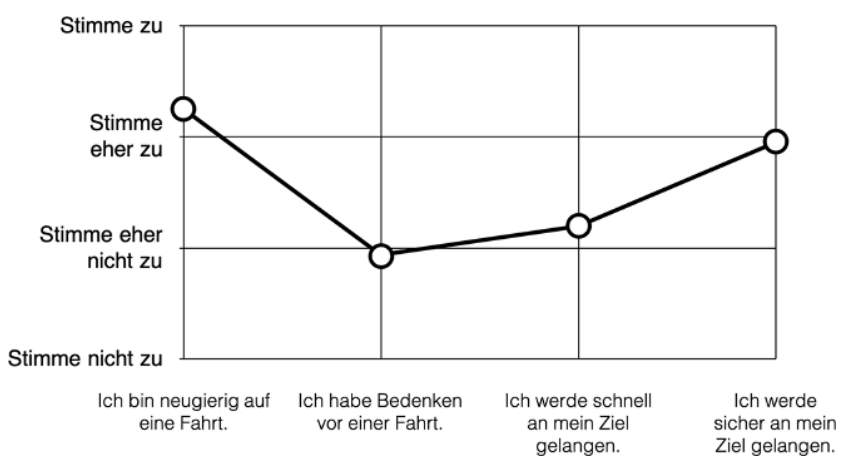

Abb. 5 Einschätzungen zur Mitfahrt schnittsgeschwindigkeit ist nicht vergleichbar mit dem klassischen ÖPNV. Die Befragung enthielt Bilder mit typischen Streckensituationen, zu denen eine Wunschgeschwindigkeit angegeben werden konnte. Die Ergebnisse legen die Abhängigkeit zwischen Wunschgeschwindigkeit, dem StraBenquerschnitt und der Verkehrssituation nahe (Abb. 6). Wobei für so gut wie jede Situation eine Geschwindigkeit präferiert wird, die sich mit der aktuellen Fahrzeuggeneration nicht erreichen lässt.

\section{Sozialer Einfluss}

Der soziale Einfluss wird hier abgebildet über den Einfluss nahestehender Personen sowie über die Bedeutung der Anwesenheit von Personal, welches die Fahrt begleitet. Die Verwendung des Shuttles durch nahestehende Personen wirkt sich positiv auf die Bereitschaft der eigenen Nutzungsintention aus. Die Befragten sind bereit, das Shuttle mit anderen Personen zu teilen - sowohl mit fremden, genauso wie mit bekannten Personen (Abb. 7).

\section{Gesellschaftliches Narrativ}

Das gesellschaftliche Narrativ thematisiert eine Bedeutungsaufladung des Shuttlebetriebs auf der Makroebene. Auch wenn der hier gewählte Ansatz ein solches Konstrukt schwer erfassen kann, wird ein gesellschaftlicher Einfluss auf die Meinungsbildung des Individuums unterstellt. Dafür wurde nach der Bedeutung der Shuttles für den ÖPNV sowie einer Einschätzung hinsichtlich der Wirkungen auf die Verkehrssituation gefragt (Abb. 8).

Übergreifend lässt sich festhalten, dass die zukünftige Bedeutung und eine einhergehende Verbesserung individueller Mobilitätsmöglichkeiten, ebenso wie Effekte auf Lärm- und Abgasemissionen, aus der Perspektive der Befragten als eher positiv bewertet werden. Erwartet wird ein besseres Angebot im ÖPNV. Wenig überzeugt sind die Befragten davon, dass fahrerlose Shuttles bestehende Verkehrsprobleme lösen können. Dementsprechend wird der positive Einfluss auf die Verhältnisse im Straßenverkehr als eher gering eingeschätzt: Kaum erwartet werden Effekte hinsichtlich der Entschärfung angespannter Verkehrssituationen oder des Raumangebots für den nichtmotorisierten Individualverkehr.

\section{Theoriediskussion: Modell zur Akzeptanz fahrerloser Shuttles}

Die folgenden Überlegungen basieren überwiegend auf einer Zusammenführung der Vorschläge von Lenz und Fraedrich (2015) sowie Rauh et al. (2020). Sie dienen der weiteren theoretischen Fundierung der Akzeptanz fahrer- 
Abb. 6 Wunschgeschwindigkeiten bei verschiedenen Straßenquerschnitten - Kronach (a) und Hof (b)
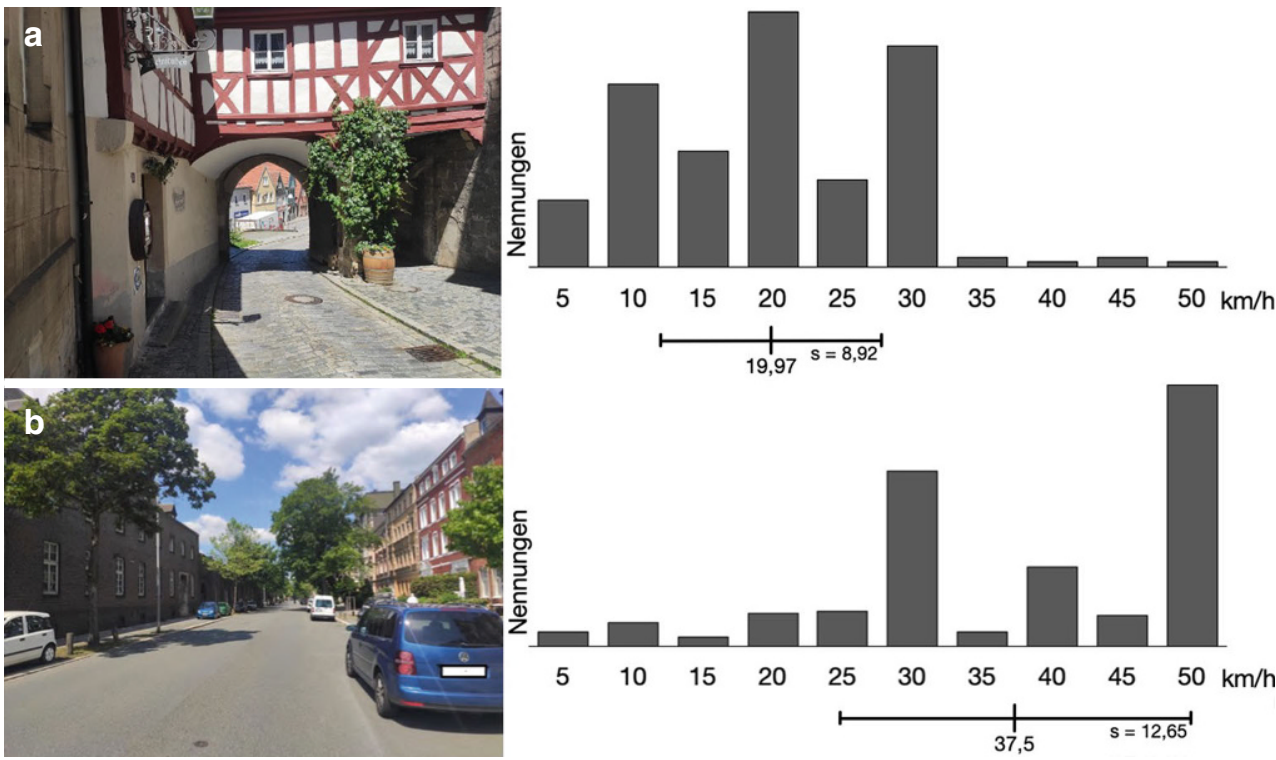

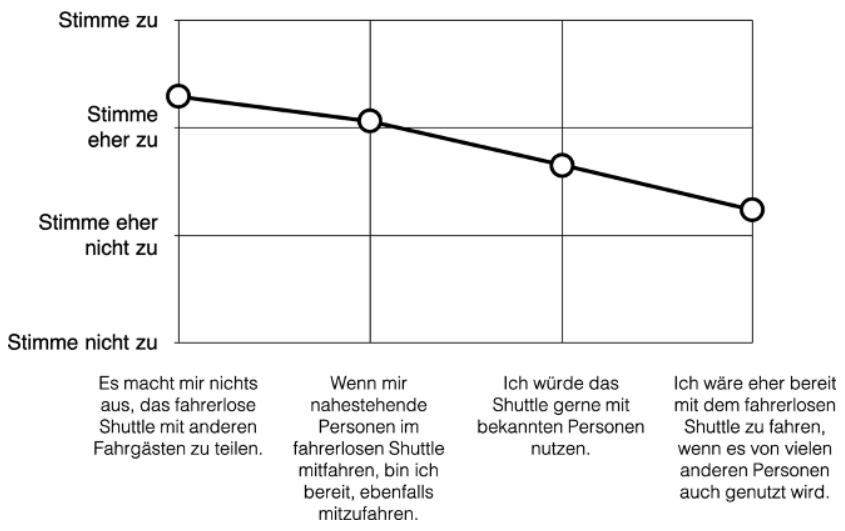

Abb. 7 Einschätzung des sozialen Einflusses in Bezug auf die Fahrt mit anderen Personen

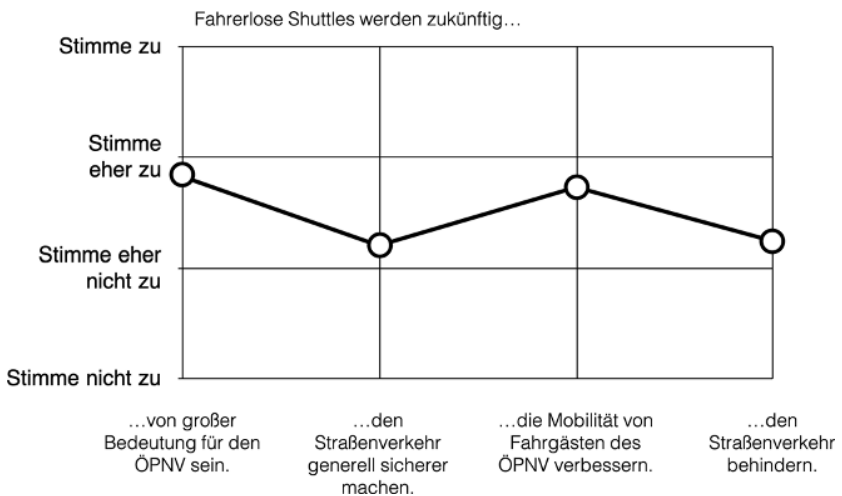

Abb. 8 Einschätzung zur Bedeutung fahrerloser Shuttles loser Shuttles. Dabei steht die Annahme im Zentrum, dass sich Akzeptanz in einem Spannungsfeld aus Subjekt, Objekt und Kontext konstituiert. Insofern setzt sich das hier vorgeschlagene Akzeptanzmodell aus 2 Dimensionen zusammen, welche Akzeptanz und Nutzungsbereitschaft beschreiben: (a) die auf das Individuum bezogene Dimension und (b) die gesellschaftliche Dimension. Die 2 Dimensionen bestehen wiederum aus erklärenden Kategorien, die die steuernden Faktoren der Akzeptanz wiedergeben - dazu zählen der soziale Einfluss, Persönlichkeitsmerkmale oder individuelle Bedeutungszuschreibungen (Abb. 9).

\section{Dimension bezogen auf das Individuum}

Die Akzeptanztheorie geht davon aus, dass das Individuum - als Akzeptanzsubjekt umschrieben - eigene Vorstellungen gegenüber dem sogenannten Akzeptanzgegenstand entwickelt und sie mit Handlungsintentionen verbindet. Der hier vorgeschlagene Ansatz beschreibt die individuellen Vorstellungen in Form von explizitem und implizitem Wissen - ein Wissen um die Technik gepaart mit der Einschätzung, welchen Einfluss der Shuttlebetrieb auf die eigene Alltagsmobilität ausübt. Zusammen mit den vorgelagerten Interessen, der eigenen Verkehrsmittelnutzung sowie den damit verbundenen Routinen (zusammengefasst als Persönlichkeitsmerkmale) beeinflusst das Wissen um die Technik die individuellen Bedeutungszuschreibungen.

Die individuellen Bedeutungszuschreibungen werden sowohl vom gesellschaftlichen Narrativ als auch vom sozialen Einfluss geprägt, genauso wie die Persönlichkeitsmerkmale und das Wissen des Einzelnen sie ausformen. Insofern kommt in den Bedeutungszuschreibungen die gesellschaftliche mit der individuellen Dimension zusammen. Die Konfiguration der Faktoren innerhalb der Bedeutungszuschrei- 


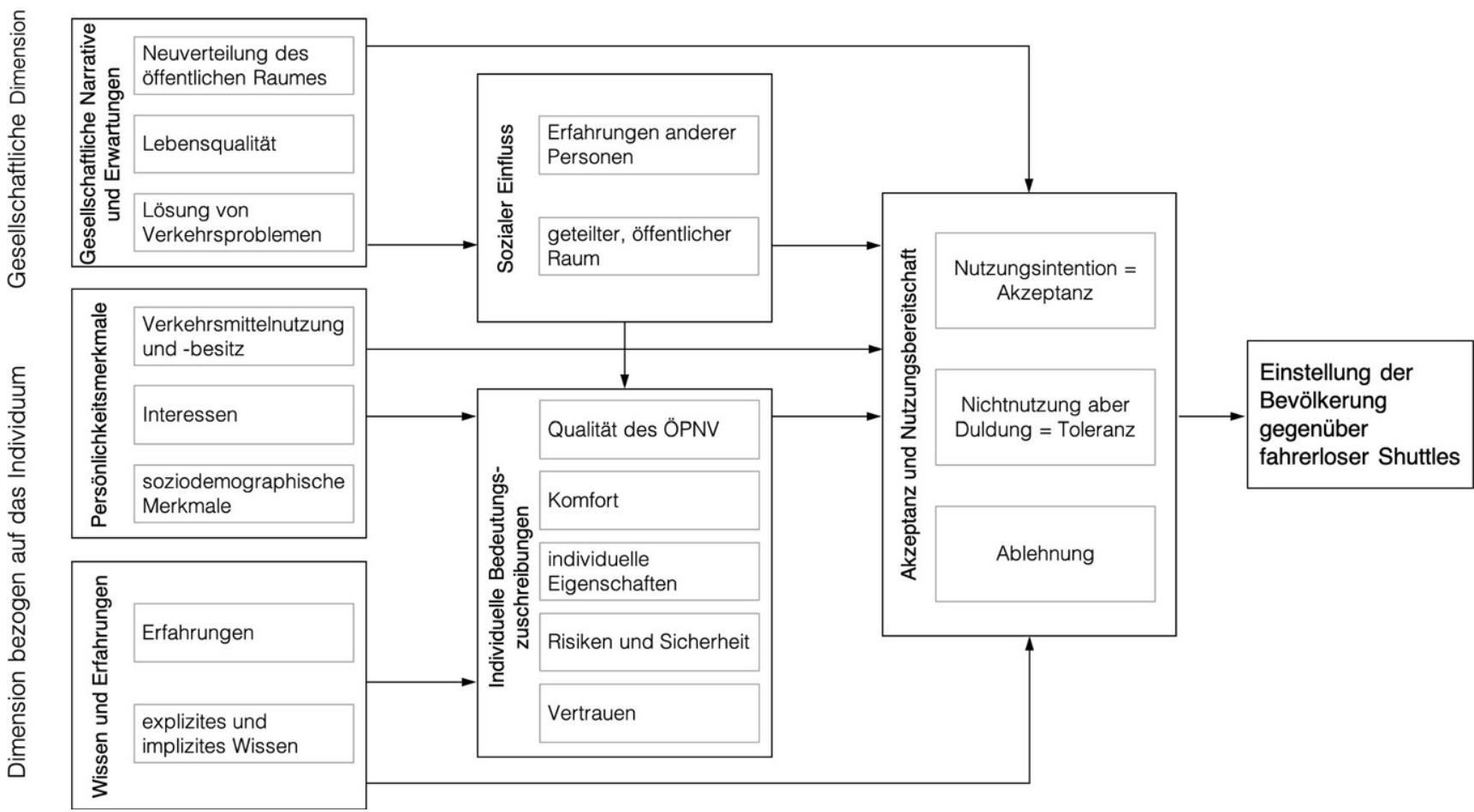

Abb. 9 Akzeptanzmodell - Einstellung der Bevölkerung gegenüber fahrerlosen Shuttles

bung drückt sich aus in der emotionalen Haltung gegenüber fahrerlosen Systemen und dem Vertrauen in deren Technik.

\section{Gesellschaftliche Dimension}

Die gesellschaftliche Dimension spannt einen Akzeptanzraum auf, in dem sich das Individuum mit seinen Eigenheiten verortet. Die Dimension wird durch gesellschaftliche Narrative ausgebildet - womit Diskurse angesprochen sind, die Werte und Normen transportieren. Sie entfalten eine Bedeutung, weil sie bewusst wie unbewusst in den Prämissen der Individuen verankert sind und so deren Handlungen beeinflussen.

Darüber hinaus ergibt sich die gesellschaftliche Dimension aus den Wertvorstellungen des sozialen Umfelds. Auch öffentlicher Raum mit seinen vorfindbaren Gegebenheiten kann als gesellschaftliches Konstrukt verstanden werden. Beides informiert die Bedeutungszuweisungen auf individueller Ebene und beeinflusst die Akzeptanz der neuen Fortbewegungsmittel.

\section{Einstellung der Bevölkerung gegenüber fahrerlosen Shuttles}

Die Ausprägungen der Faktoren formen die Akzeptanz und Nutzungsbereitschaft fahrerloser Shuttles. Dazu können 3 Typen beschrieben werden:
1. Nutzungsintention =Akzeptanz: Die Akzeptanz gegenüber der Technik und dem Betrieb schlägt sich als Nutzungsintention nieder. Die Menschen sind bereit, die Technik auszuprobieren und sind dem Betrieb grundsätzlich positiv gegenüber eingestellt.

2. Nichtnutzung aber Duldung = Toleranz: Zwar werden mit dem Betrieb keine negativen Auswirkungen verbunden, ein persönlicher Nutzen wird allerdings auch nicht erwartet. Die Haltung lässt sich als Duldung umschreiben: prinzipiell aufgeschlossen, aber frei von einer Nutzungsintention.

3. Ablehnung: Sowohl Technik als auch der Betrieb wird mit negativen Eigenschaften belegt. Diese symbolische Aufladung führt zur Ablehnung fahrerloser Shuttles.

Die 3 Kategorien stehen nicht trennscharf zueinander. So bestätigen die vorliegende wie auch andere Arbeiten, dass auf der einen Seite fahrerlose Systeme assoziiert werden mit individuellen und gesellschaftlichen Vorteilen, wohingegen auf der anderen Seite eine skeptische Haltung gegenüber der Technik eingenommen wird (vgl. Battistini et al. 2020).

\section{Schlussbetrachtungen}

In den 3 SMO-Kommunen besteht bereits vor dem Betriebsbeginn eine ausgeprägte Bereitschaft in der Bevölkerung, die fahrerlosen Shuttles zu nutzen. Dabei legte die Erhe- 
bung eine Erwartungshaltung offen: Die Menschen verbinden mit den fahrerlosen Shuttles flexible Abfahrten und eine hohe Taktdichte, Pünktlichkeit und neue Strecken, bessere Erreichbarkeit und Angebote in Randzeiten. Eine überhöhte symbolische Aufladung deckt sich mit den Ergebnissen anderer Studien (Canzler und Knie 2019, S. 17).

Die Erwartungen spiegeln sich bislang kaum in der Leistungsfähigkeit der aktuellen Generation fahrerloser Shuttles wider. Obwohl fortgeschritten, befinden sich sowohl Technik als auch die Integration in den ÖPNV in einem Anfangsstadium. Die Diskrepanz zwischen gesellschaftlichem Narrativ, Erwartungshaltung und Leistungsfähigkeit kann die Akzeptanz in der Bevölkerung gefährden. Begünstigt durch eine auf gesellschaftlicher Ebene verortete symbolische Aufladung lässt sich eine gewisse Euphorie gegenüber der Technik unterstellen. Sollten Erwartungen unerfüllt bleiben, kann eine Ernüchterung in der Bevölkerung eintreten. Ernüchterung führt wiederum dazu, dass die bestehende Akzeptanz umschlägt in Duldung oder sogar Ablehnung.

Der Realbetrieb fahrerloser Shuttles befindet sich in der Anfangsphase der Diffusion - wie sie sich entfalten wird, ist schwer einzuschätzen. Um die insgesamt hohe Akzeptanz in der Bevölkerung auf diesem Niveau zu halten, bedarf es eines geschärften Blicks auf die Zusammenhänge zwischen gesellschaftlichem Narrativ, individuellen Bedeutungszuschreibungen und der Leistungsfähigkeit des Systems.

Förderung Das SMO Projekt ist gefördert durch das Bundesministerium für Verkehr und digitale Infrastruktur. Für Informationen zum Projekt siehe: www.shuttle-modellregion-oberfranken.de.

Funding Open Access funding enabled and organized by Projekt DEAL.

Open Access Dieser Artikel wird unter der Creative Commons Namensnennung 4.0 International Lizenz veröffentlicht, welche die Nutzung, Vervielfältigung, Bearbeitung, Verbreitung und Wiedergabe in jeglichem Medium und Format erlaubt, sofern Sie den/die ursprünglichen Autor(en) und die Quelle ordnungsgemäß nennen, einen Link zur Creative Commons Lizenz beifügen und angeben, ob Änderungen vorgenommen wurden.

Die in diesem Artikel enthaltenen Bilder und sonstiges Drittmaterial unterliegen ebenfalls der genannten Creative Commons Lizenz, sofern sich aus der Abbildungslegende nichts anderes ergibt. Sofern das betreffende Material nicht unter der genannten Creative Commons Lizenz steht und die betreffende Handlung nicht nach gesetzlichen Vorschriften erlaubt ist, ist für die oben aufgeführten Weiterverwendungen des Materials die Einwilligung des jeweiligen Rechteinhabers einzuholen.

Weitere Details zur Lizenz entnehmen Sie bitte der Lizenzinformation auf http://creativecommons.org/licenses/by/4.0/deed.de.

\section{Literatur}

Battistini R, Mantecchini L, Postorino MN (2020) Users' acceptance of connected and automated shuttles for tourism purposes: a survey study. Sustainability 12:10188. https://doi.org/10.3390/ su122310188

Bernhard C, Oberfeld D, Hoffmann C et al (2020) User acceptance of automated public transport. Transp Res Part F Traffic Psychol Behav 70:109-123. https://doi.org/10.1016/j.trf.2020.02.008

Canzler W, Knie A (2019) Autonom und Öffentlich: Automatisierte Shuttles für mehr Mobilität mit weniger Verkehr https://doi.org/ $10.25530 / 03552.39$

Chen C-F (2019) Factors affecting the decision to use autonomous shuttle services: evidence from a scooter-dominant urban context. Transp Res Part F Traffic Psychol Behav 67:195-204. https://doi. org/10.1016/j.trf.2019.10.016

Kassens-Noor E, Kotval-Karamchandani Z, Cai M (2020) Willingness to ride and perceptions of autonomous public transit. Transp Res Part A Policy Pract 138:92-104. https://doi.org/10.1016/j.tra. 2020.05.010

Lenz B, Fraedrich E (2015) Gesellschaftliche und individuelle Akzep$\operatorname{tanz}$ des autonomen Fahrens. In: Autonomes Fahren. Springer, Berlin, Heidelberg, S 639-660

Nordhoff S, Louw T, Innamaa S et al (2020) Using the UTAUT2 model to explain public acceptance of conditionally automated (L3) cars. Transp Res Part F Traffic Psychol Behav 74:280-297. https://doi. org/10.1016/j.trf.2020.07.015

Paddeu D, Parkhurst G, Shergold I (2020) Passenger comfort and trust on first-time use of a shared autonomous shuttle vehicle. Transp Res Part C Emerg Technol 115:102604. https://doi.org/10.1016/j. trc. 2020.02 .026

Rauh J, Appel A, Graß1 M (2020) Empirische Beobachtungen zur Akzeptanz des Pilotprojektes „Autonom fahrender Kleinbus“ unter den Bürger*innen von Bad Birnbach. In: Riener A et al (Hrsg) Autonome Shuttlebusse im ÖPNV. Springer, Berlin Heidelberg, S $159-176$

Rosell J, Allen J (2020) Test-riding the driverless bus: determinants of satisfaction and reuse intention in eight test-track locations. Transp Res Part A Policy Pract 140:166-189. https://doi.org/10. 1016/j.tra.2020.08.013

Venkatesh V, Morris MG, Davis GG, Davis FD (2003) User acceptance of information technology: toward a unified view. MISQ 27:425. https://doi.org/10.2307/30036540 